\title{
Interferon-gamma release assay for detection of latent tuberculosis infection in casual and close contacts of tuberculosis cases
}

A.M.F. Abu-Taleb, ${ }^{1}$ R.H. El-Sokkary ${ }^{1}$ and S.A. El Tarhouny ${ }^{2}$

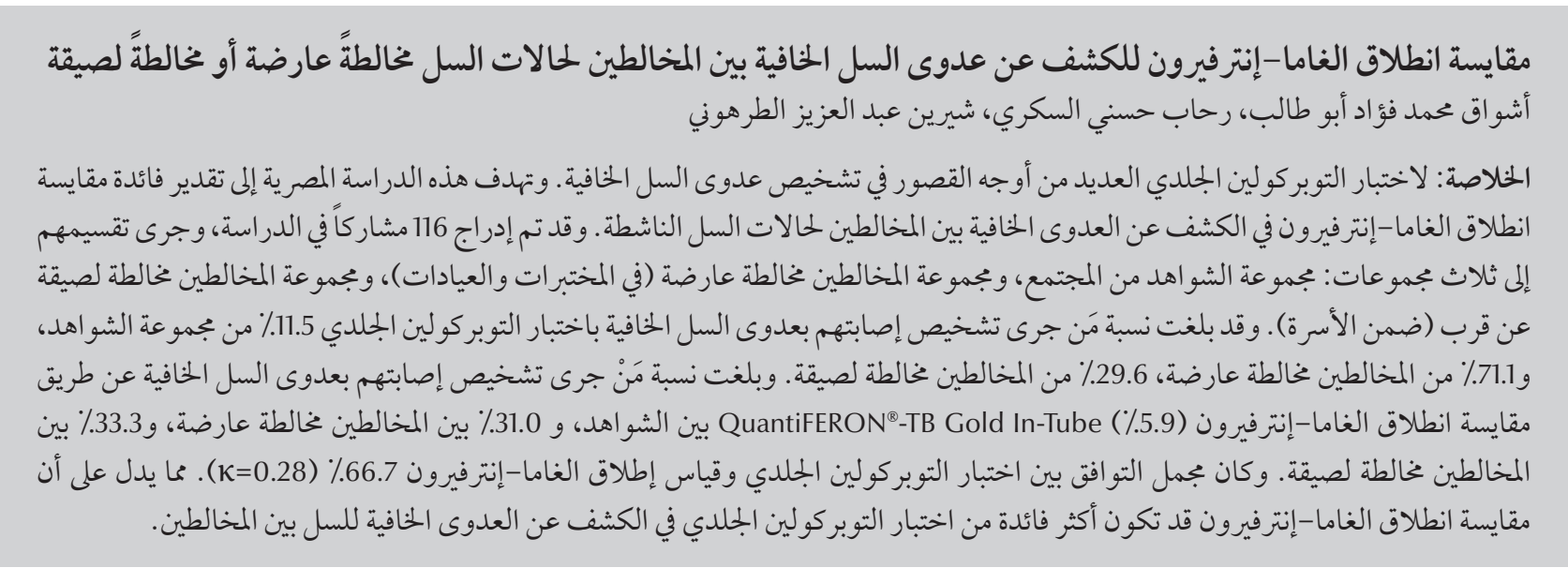

ABSTRACT The tuberculin skin test (TST) has many limitations for the diagnosis of latent tuberculosis (TB) infection. The aim of this study in Egypt was to estimate the usefulness of an interferon-gamma release assay (IFN- $\gamma$ ) assay for the detection of latent TB infection in contacts of active TB cases. A total of 116 participants were enrolled and divided into 3 groups: community controls, casual (laboratory and clinic) contacts and close (household) contacts. Subjects diagnosed with latent TB infection by TST were $11.5 \%$ of controls, $71.1 \%$ of casual contacts and $29.6 \%$ of close contacts. Subjects diagnosed as latent TB infection by IFN- $\gamma$ assay (QuantiFERON ${ }^{\circledR}-$ TB Gold In-Tube) were $5.9 \%$ of controls, $31.0 \%$ of casual contacts and $33.3 \%$ of close contacts. The overall agreement between TST and IFN- $\gamma$ was $66.7 \%(\kappa=0.28)$. The IFN- $\gamma$ method could be more helpful than TST for detection of latent TB infection in contacts.

Test de quantification de la libération d'interféron gamma pour le dépistage des infections tuberculeuses latentes chez les contacts occasionnels et rapprochés des patients atteints de tuberculose

RÉSUMÉ Le test tuberculinique cutané comporte de nombreuses limitations pour le diagnostic d'une infection tuberculeuse latente. L'objectif de la présente étude conduite en Égypte était d'évaluer l'utilité d'un test de quantification de la libération d'interféron gamma (IFN- $\gamma$ ) pour le dépistage des infections tuberculeuses latentes chez les contacts de patients atteints de tuberculose active. Au total, 116 participants ont été recrutés et répartis en trois groupes : les témoins communautaires, les contacts occasionnels (laboratoires et établissements de soins) et les contacts rapprochés (vivant sous le même toit). Les sujets ayant reçu le diagnostic d'infection tuberculeuse latente aux moyens du test tuberculinique cutané représentaient 11,5\% du groupe témoin, 71,1 \% du groupe des contacts occasionnels et $29,6 \%$ du groupe des contacts rapprochés. Les sujets ayant reçu le diagnostic d'infection tuberculeuse latente aux moyens du test IFN- $\gamma$ (QuantiFERON ${ }^{\circledR}$-TB Gold In-Tube ${ }^{\circledR}$ ) représentaient 5,9\% du groupe témoin, $31,0 \%$ du groupe des contacts occasionnels et 33,3\% du groupe des contacts rapprochés. La concordance globale entre les deux tests s'élevait à $66,7 \%(\kappa=0,28)$. La méthode du test IFN- $\gamma$ pourrait être plus utile que le test tuberculinique cutané pour le dépistage des infections tuberculeuses latentes chez les sujets contacts.

${ }^{7}$ Department of Medical Microbiology and Immunology; ${ }^{2}$ Department of Medical Biochemistry, Faculty of Medicine, University of Zagazig, Zagazig, Egypt (Correspondence to.H. El-Sokkary: rehab_elsokkary@yahoo.com).

Received: 12/10/09; accepted: 18/03/10 


\section{Introduction}

Globally, about 2 billion people are infected with Mycobacterium tuberculosis; 8 to 10 million develop active disease and 2 million die from tuberculosis (TB) every year [1]. In Egypt, the incidence of TB is estimated at 21 per 100000 population per year [2]. One of the first priorities of TB control programmes is the identification of people with latent TB infection [3] in order to prevent development of active disease [4]. Accurate diagnosis of latent TB infection is scientifically challenging, however, because of the low burden of dormant tubercle bacilli, which are not directly detectable or quantifiable. Besides, latent TB infection is asymptomatic, with patients having no clinical or radiographic signs of active TB yet carrying a small but significant risk of progression to active $\mathrm{TB}[5]$.

Until recently, the tuberculin skin test (TST), which uses purified-protein derivative (PPD), was the only method available for the diagnosis of latent TB infection [5]. The TST has many limitations, including the need for at least 2 patient visits, problems in administration, problems in interpretation and difficulty in separating true infection from the effects of prior bacille CalmetteGuérin (BCG) vaccination and infection by non-tuberculous Mycobacteria spp. [6]. Despite these drawbacks, TST remains in widespread use because of its advantages, being inexpensive, simple and practical in many settings) [7].

Advances in genomics and immunology have led to a promising alternative to TST: in vitro interferon-gamma (IFN- $\gamma$ ) release assays. These tests are based on the principle that T-cells of individuals infected with $M$. tuberculosis bacilli release IFN- $\gamma$ when they re-encounter TB-specific antigens [8]. The QuantiFERON ${ }^{\circ}$-TB assay is of these tests. The latest generation of these tests - the QuantiFERON-TB ${ }^{\circ}$ Gold In-Tube method (QFT Gold-IT) has increased specificity for detection of $M$. tuberculosis infection due to the use of 3 specific antigens. It requires a single patient visit, the interpretation is less subjective than the TST and the test is not affected by prior BCG vaccination or infection by most non-TB Mycobacteria [9].

The aim of this study was to estimate the usefulness of an IFN- $\gamma$ assay for the detection of latent $\mathrm{TB}$ infection in contacts of active TB cases.

\section{Methods}

This was a comparative cross-sectional study conducted in the Department of Medical Microbiology and Immunology, Faculty of Medicine, University of Zagazig, Egypt. The study was conducted over 7 months from August 2008 to February 2009.

\section{Participants}

Clinically suspected TB patients were selected by systemic random sampling from those attending outpatient clinics in Zagazig Chest Hospital and Chest Department in Zagazig University Hospitals during the study period. All those with bacteriological confirmation of the diagnosis were used as the index cases. Three groups of people were selected for the study according to their degree of exposure to the index cases (on the assumption that contact either directly with TB patients or with specimens is a risk factor $[10,11])$.

- Close contacts: healthy individuals with a history of close contact with a TB patient for more than 8 hours per day, i.e. household contacts. We selected 2 contacts by systemic random sampling from all household contacts of confirmed TB cases.

- Casual contacts: health care workers with a history of casual contact with TB cases or specimens. They were recruited from among medical staff members and laboratory technicians who were on duty during the study period. Employees with a history of TB were excluded.

- Community controls: healthy individuals without an identified risk for M. tuberculosis exposure. These were recruited from employees in other departments of the Faculty of Medicine.

By using data from the records, people were excluded if they had symptoms suggestive of active $\mathrm{TB}$, showed abnormal chest radiographs or had history of active TB. All participants were selected by simple random sampling.

The sample size was determined assuming a prevalence of TB infection in Egypt of 27 per 100000 per year (based on World Health Organization data [2]) and non-response rate of $20 \%$ (based on previous studies in the same locality and the same population [12]) and using $\kappa$ coefficient $>0.75$; $\alpha$-error $=0.05$ and $\beta$-error $=0.20$. Using these parameters and assumptions, and Epiinfo, version 9 software, the minimum sample size for each group was estimated to be 25 .

\section{Data collection}

After giving their informed written consent, participants were interviewed and asked about their age, sex, history of prior TB disease and history of exposure to TB cases.

All groups were subjected to TST and INF- $\gamma$ assay as follows. TST was performed by using $0.1 \mathrm{~mL}$ of 5 tuberculin units PPD (Vacsera), and interpretation of the results was done according to American Thoracic Society guidelines [6]. For the INF- $\gamma$ assay a blood sample was collected into 3 evacuated tubes, the first tube coated with TB-specific antigens (ESAT-6, CFP10 and TB7.7), the second tube coated with heparinnegative control and the third tube coated with mitogen (phytohaemagglutinin) as positive control. Blood was incubated overnight at $37^{\circ} \mathrm{C}$. Plasma was then separated to measure the concentration of IFN- $\gamma$ by enzyme-linked immunosorbent assay using the QFT- 
Gold IT assay (Cellestis) following the manufacturer's instructions. The cut-off value for positive findings was $\geq 0.35$ $\mathrm{IU} / \mathrm{mL}[13]$.

Any cases of latent TB diagnosed were referred to the corresponding clinician for further management.

\section{Statistical analysis}

Concordance between the test results from the TST and the QFT-Gold IT assay was assessed using kappa coefficients $(\kappa=0.75$ excellent agreement; $\kappa=$ 0.40 poor agreement; and $\kappa=0.40-0.75$ fair to good agreement). We measured the correlation of the 2 tests with the risk of latent TB infection by estimating the odds ratio (OR) and 95\% confidence intervals $(\mathrm{CI})$ relating the test results to the likelihood of TB infection. Data were analysed using SPSS, version 11 software package.

\section{Results}

A total of 116 participants were included in this study: 28 household contacts of TB cases (12 males and 16 females); 48 health care workers (16 males and 32 females) comprising 30 medical staff members and 18 laboratory technicians; and 40 controls ( 13 males and 27 females). The median and range of ages of the groups are show in Table 1.

A documented TST result was obtained in 91 (78.5\%) of the 116 participants; 8 did not attend the follow-up reading of the test, 5 refused to perform it fearing pain and 12 refused to be injected by foreign material. There were no significant differences in the personal and medical characteristics of the refusers and active participants. Of the 91 participants tested, 38 (41.8\%) were positive for TST. In the close contacts group there were $8 / 27$ (29.6\%) positive cases ( 4 males and 4 females, age range 15-60 years). In the casual contacts group there were $27 / 38(71.1 \%)$ positive cases (11 males and 16 females, age range $2-55$ years). In the control group there were $3 / 26(11.5 \%)$ positive cases ( 1 male and 2 females, age range 28-70 years) (Table 2).

A valid QFT-Gold IT assay result was obtained in 100 of the 116 participants; 16 readings were indeterminate and were excluded from further analysis. Among the 100 valid results, there were 23 (23.0\%) positive cases. In the close contacts group there were $8 / 24$ (33.3\%) positive cases ( 4 males and 4 females, age range 2-55 years) and in the casual contacts group there were $13 / 42(31.0 \%)$ positive cases (4 males and 9 females, age range $20-55$ years). In the control group there were $2 / 34$ (5.9\%) positive cases (1 male and 1 female, age range 33-70 years) (Table 2).

There were 75 subjects with valid (i.e. determinate) results for both TST and QFT-Gold IT. They were distributed as follows: 20 controls, 32 casual contacts and 23 close contacts. The concordance between both tests within different subgroups is shown in Table 3. Using the TST, the risk of infection appeared to be greatly increased in the casual contacts group (OR $=18.8,95 \%$ CI: 4.1-99.5, $P<0.001)$ compared with the close contacts group $(\mathrm{OR}=3.2$, 95\% CI: $0.6-8.1, P=0.1)$. With the QFT-Gold IT assay, the increased the risk of infection with exposure was similar in the casual contacts group $(\mathrm{OR}=$ 7.2, 95\% CI: $1.4-50.5, P=0.006)$ and close contacts group (OR = 8.0, 95\% CI: 1.3-62.4, $P=0.01)$ (Table 2).

\section{Discussion}

In high-burden countries, the diagnosis and treatment of active $\mathrm{TB}$ receives greater priority; testing for latent TB infection is usually done only in selected high-risk groups. Traditionally, latent TB infection has been diagnosed with a TST. However, the TST has limitations. The antigens present in PPD and used for the TST are also present in the BCG vaccine strain and therefore false positive tests are common. This has led to the development of novel tests of latent TB infection that use $M$. tuberculosis-specific antigens [14]. So in the present study we aimed to estimate the usefulness of an IFN- $\gamma$ assay for the rapid detection of mycobacterial $\mathrm{TB}$ infection in individuals in contact with TB cases or specimens.

In the control group, participants diagnosed as latent TB infection were $11.5 \%$ by TST and $5.9 \%$ by IFN- $\gamma$. Our findings agree with those of Porsa et al. in the United States, who found latent

\begin{tabular}{|c|c|c|c|c|}
\hline Variable & $\begin{array}{l}\text { Community controls } \\
(n=40)\end{array}$ & $\begin{array}{l}\text { Casual contacts } \\
\qquad(n=48)\end{array}$ & $\begin{array}{l}\text { Close contacts } \\
\quad(n=28)\end{array}$ & $P$-value \\
\hline \multicolumn{5}{|l|}{ Age (years) } \\
\hline Mean (SD) & $41.9(16.2)$ & $36.4(9.2)$ & $22.5(16.7)$ & \\
\hline Median (range) & 33.5 (25-76) & $36.0(18-55)$ & $19.5(2-55)$ & $<0.001$ \\
\hline \multicolumn{5}{|l|}{ Sex [no. (\%)] } \\
\hline Male & $13(32.5)$ & $16(33.3)$ & $12(42.9)$ & $>0.5$ \\
\hline Female & $27(67.5)$ & $32(66.7)$ & $16(57.1)$ & \\
\hline
\end{tabular}

$S D=$ standard deviation . 


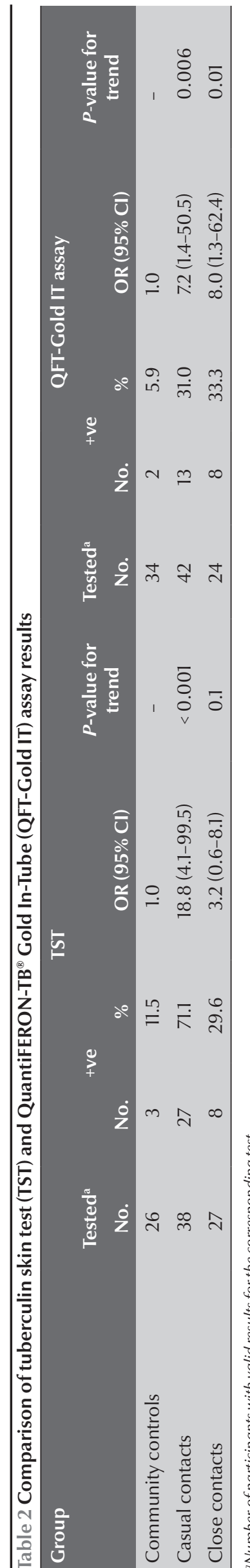

TB in $9.0 \%$ and $5.4 \%$ of subjects by TST and IFN- $\gamma$ respectively [15].

In our casual contacts group, $71.1 \%$ of subjects were diagnosed as latent $\mathrm{TB}$ infection by TST and $31.0 \%$ by IFN- $\gamma$ assay. This discrepancy might be explained by a high rate of false positives for the TST. Health care workers in our faculty receive repeated BCG vaccination and also repeat TSTs during their employment. These 2 factors may further boost their reaction to the TST and thus lead to a false positive TST result [16]. This hypothesis is supported by the results of many previous studies [17].

In our close contacts group, $29.6 \%$ of subjects were diagnosed as latent $\mathrm{TB}$ infection by TST and $33.3 \%$ by IFN- $\gamma$ assay. This disagrees with the Korean study of Kang et al., in which $71 \%$ of subjects were diagnosed as latent $\mathrm{TB}$ infection by TST and $44 \%$ by IFN- $\gamma$ assay. This difference might also be explained by a higher rate of false positive results in their study for TST than in ours due to different vaccination schedules. In Korea, $B C G$ vaccination is given at birth and again at age 12 or 13 years if the child proves to be a TST non-responder [18]. In Egypt, on the other hand, BCG vaccination is given in infancy, which is unlikely to affect TST reactions in adolescence or adult life [19].

Our study showed a poor correlation between TST and IFN- $\gamma$ among the investigated groups. The overall agreement was $66.7 \%(\kappa=0.28)$, which is similar to the findings of Mahomed et al. in South Africa, who found agreement of $69 \%(\kappa=0.32)$ [9].
As there is no gold standard for the diagnosis of latent TB infection the measurement of correlation with the risk of infection could be a more sensitive method to compare the utility of the TST and IFN- $\gamma$ than a detailed determination of the specificity and sensitivity of tests. In our study, the TST results showed that TB exposure increased the risk of infection 18.8-fold in the casual contacts group and 3.2-fold in the close contacts group. The IFN- $\gamma$ results showed that exposure increased the risk of infection 7.2-fold in the casual contacts group and 8-fold in the close contacts group and this was in agreement of the findings of Kang et al. [18].

Although our study showed better results with the IFN- $\gamma$ assay, we should consider the relatively higher costs, practical inconvenience and the presence of indeterminate test results. By conducting a preliminary estimation of the cost of QFT-Gold IT to process a sample from 1 patient, the cost would be between 250 and 300 EGP (Egyptian pounds) while the cost of the TST is as low as 15 and 20 EGP (US\$ $1=6 \mathrm{EGP})$. In addition, for the QFTGold IT, at least 22 patients should be analysed per run so as not to waste wells and therefore the minimum sample costs will only be achieved when all wells are used.

In conclusion, the IFN- $\gamma$ assay showed better performance than the TST in subjects with latent TB infection and it correlated better with exposure to M. tuberculosis. An IFN- $\gamma$ assay might help to avoid the limitations of

\begin{tabular}{|c|c|c|c|}
\hline \multicolumn{4}{|l|}{$\begin{array}{l}\text { Table } 3 \text { Concordance } \\
\text { In-Tube assay results }\end{array}$} \\
\hline \multirow[t]{2}{*}{ Group } & $\begin{array}{l}\text { Observed } \\
\text { agreement }\end{array}$ & Coefficient & $P$-value \\
\hline & $\%$ & א-value (SE) & \\
\hline Community controls & 95.0 & $0.77(0.21)$ & $<0.001$ \\
\hline Casual contacts & 40.6 & $0.02(0.12)$ & 0.56 \\
\hline Close contacts & 78.2 & $0.50(0.20)$ & 0.007 \\
\hline All & 66.7 & $0.28(0.10)$ & 0.004 \\
\hline
\end{tabular}

$S E=$ standard error. 
the TST in BCG-vaccinated populations as it uses antigens more specific to $M$. tuberculosis than the PPD used in the TST, and prior BCG vaccination does not affect the results. We recommend that the QFT-Gold IT could be more helpful than the TST for the detection of latent TB infection despite the risk of underestimation of cases.

\section{Acknowledgements}

The authors are grateful to Professor Ahmed-Refat Refat, Professor of
Occupational Medicine, Faculty of Medicine, University of Zagazig for his helpful remarks and valuable assistance.

This work was funded by support of academic research in Zagazig University Projects. University of Zagazig Postgraduate and Research Affairs

\section{References}

1. Global tuberculosis control: surveillance, planning, financing. WHO report. Geneva, World Health Organization, 2006 (WHO/HTM/TB/2006.362).

2. Global tuberculosis control: epidemiology, strategy, financing. WHO report. Geneva, World Health Organization, 2009.

3. Broekmans JF et al. European framework for tuberculosis control and elimination in countries with a low incidence. Recommendations of the World Health Organization (WHO), International Union Against Tuberculosis and Lung Disease (IUATLD) and Royal Netherlands Tuberculosis Association (KNCV) Working Group. European Respiratory Journal, 2002, 19:765-775.

4. Anderson P et al. Specific immune-based diagnosis of tuberculosis. Lancet, 2000, 356:1099-1104.

5. Lalvani A. Diagnosing tuberculosis infection in the 21st century. New tools to tackle an old enemy. Chest, 2007, 131:18981906.

6. American Thoracic Society. Targeted tuberculin testing and treatment of latent tuberculosis infection. American Journal of Respiratory and Critical Care Medicine, 2000, 161:S221-S247.

7. Clinical guideline 33. Tuberculosis: clinical diagnosis and management of tuberculosis and measurement for its prevention and control. London, National Institute for Health and Clinical Excellence, 2006.

8. Lalvani A, Millington KA. T-cell interferon- release assays: can we do better? European Respiratory Journal, 2008, 32:14281430 .

9. Mahomed $\mathrm{H}$ et al. Comparison of Mantoux skin test with three generations of a whole blood IFN- $\gamma$ assay for tuberculosis infection. International Journal of Tuberculosis and Lung Disease, 2006, 10:310-316.

10. Challu VK. Safety in TB research laboratory. NTI Bulletin, 2005, 41:97-100.
11. Guidelines for preventing the transmission of Mycobacterium tuberculosis in health-care settings, 2005. Morbidity and Mortality Weekly Report, 2005, 54 (RR-17).

12. Khalil GM et al. Job hazards analysis among a group of surgeons at Zagazig university hospitals: a risk management approach. Zagazig Journal of Health, 2009, 2:29-39.

13. QuantiFERON ${ }^{\circledR}$-TB Gold (in-tube method). Package insert. Cellestis [website] (http://www.cellestis.com/IRM/Company/ ShowPage.aspx?CPID=1171, accessed 15 August 2011).

14. Pai M. Alternatives to the tuberculin skin test: interferongamma assays in the diagnosis of mycobacterium tuberculosis infection. Indian Journal of Medical Microbiology, 2005, 23:151-158.

15. Porsa E et al. Comparison of a new ESAT-6/CFP-10 peptidebased gamma interferon assay and a tuberculin skin test for tuberculosis screening in a moderate-risk population. Clinical and Vaccine Immunology; CVI, 2006, 13:53-58.

16. Kang YA et al. Discrepancy between the tuberculin skin test and the whole-blood interferon gamma assay for the diagnosis of latent tuberculosis infection in an intermediate tuberculosis-burden country. Journal of the American Medical Association, 2005, 293:2756-2761.

17. Al-Jahdali $\mathrm{H}$ et al. The utility and interpretation of tuberculin skin tests in the Middle East. American Journal of Infection Control, 2005, 33:151-156.

18. Farhat $\mathrm{M}$ et al. False-positive tuberculin skin tests: what is the absolute effect of BCG and non-tuberculous mycobacteria? International Journal of Tuberculosis and Lung Disease, 2006, 10:1192-1204.

19. Lillian $\mathrm{KO}$ et al. Tuberculosis risk among nursing professionals from central Brazil. American Journal of Infection Control, 2008, 36:148-151. 